\title{
CORBA-BASED INTEROPERATION IN SUBSTATION AUTOMATION SYSTEMS ${ }^{1}$
}

\author{
Ricardo Sanz* Ignacio González ${ }^{* *}$ Miguel Segarra ${ }^{* * *}$ \\ Raoul Tagliarini ${ }^{* * * *}$ Luis Gomes ${ }^{\dagger}$ Gregorio Hernández ${ }^{\ddagger}$ \\ Antonio Zangrilli ${ }^{\S}$
}

\author{
* Universidad Politécnica de Madrid, Spain \\ ** Eliop, Spain \\ *** SCILabs Ingenieros, Spain \\ **** Decan, France \\ ${ }^{\dagger}$ Uninova, Portugal \\ ¥Red Eléctrica de España, Spain \\ $\S$ Innova, Italy
}

\begin{abstract}
Substation Automation Systems are distributed command and control systems that operate equipment in electrical substations. Traditional automation systems were built as turnkey systems based on proprietary technology but recent developments in automation and networking have created a new wave of interoperability requirements. This paper addresses some of these topics and focus on recent standardization activities in the field, in particular in relation with the incorporation of object technology in this area. In particular, it describes a real implementation of the emerging standard IEC 61850 using CORBA networking in the European R+D project DOTS.
\end{abstract}

Keywords: Substation Automation Systems, integration, distributed control, interoperation, standardization, real-time object oriented systems.

\section{INTRODUCTION}

Substation automation systems (SAS) are distributed command and control systems that operate equipment in electrical substations. Traditional systems were built as turnkey systems based on proprietary technology but recent developments in automation systems and field networking have created a new wave of interoperability requirements.

Information technology in the telecontrol field has been contributing to the cost reduction of systems, providing new software solutions and devices, but huge obstacles are still pending for the achieve-

\footnotetext{
1 This work has been done with partial funding from the European Commission and the Comisión Interministerial de Ciencia y Tecnología.
}

ment of a complete interoperability of devices, particularly due to the existence of dozens of proprietary systems and protocols. Standardization is a key issue here as it offers a common ground for interoperation across vendor boundaries. There are several ongoing standardization activities in different bodies: IEC, EPRI, IEEE, OMG.

This paper describes the results of DOTS Project. This is a research \& development project funded by the European Commission (DOTS Consortium, 1999), which offers a new technological solution for the construction of SAS that allows to overcome some integrational drawbacks by means of widely accepted object oriented specifications and protocols from OMG. 
DOTS stands for Distributed Objects Telecontrol Systems and Networks. The DOTS Consortium was composed by: Eliop (ES) that develops core automation functions; Innova (IT) as the responsible of the exploitation of the results; SCILabs Ingenieros (ES) providing a real-time, embedable ORB for the IEDs; UNINOVA (PT) develops the SAS configuration application; DECAN (FR) developing operation terminals; Universidad Politécnica de Madrid (ES) as authors of the mapping to the ACSI of IEC-61850 to CORBA; and Red Eléctrica de España (ES) is the final user, participating in the definition of the needs and providing the plant to demonstrate the project application.

\section{BRIEF DESCRIPTION}

The main objective of DOTS is the development of control system models based on emergent telecontrol standards and an open software architecture built upon real-time distributed object technologies. This technology will allow the simplified development of SAS and an optimum exploitation of the interoperation capabilities of intelligent electronic devices in the distributed context of an electric power grid.

DOTS architecture ensures interoperability among multivendors telecontrol devices through the IEC61850 standard (Communication Networks and Systems in Substations) (IEC TC-57, 2000c). The implementation is based on a mapping to the CORBA (Common Object Request Broker Architecture)(OMG, 2000) middleware. This mapping has been submitted to the IEC for inclusion as part of the the IEC 61850 standard.

The IEC 61850 standard specifies a collection of elementary services that can be performed by SAS: breaker operation, monitorization, reporting, etc. These services (referred-to as logical nodes) can be implemented using different types of technologies and are distributed in a collection of independent processors (remote terminal units, intelligent electronic devices, station computers, etc.). The global behavior of the system is achieved by means of gathering a collection of such services in global system functions. For example, to provide a Synchronized Switching function it is necessary to integrate several logical nodes: Synchronized Switching, Human-machine Interface, Breaker Control, etc.

Figure 1 shows how system functionality is provided by means of combination of elementary components. For example, to provide overcurrent protection the systems employs a Human-Machine Interface, a Protection, a Breaker and a Current Transformer logical nodes, i.e. elementary pieces of functionality that combined provide the overall function.

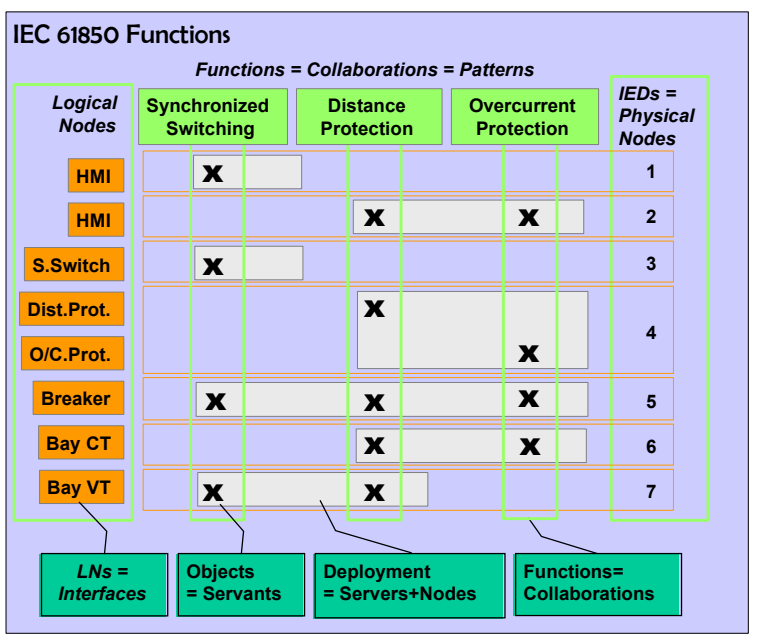

Fig. 1. Implementing system functions as collaborations of elementary services as specified by IEC 61850 and DOTS mapping to CORBA.

The figure also shown how these concepts are mapped to CORBA concepts. Logical nodes, the central element of the specification, are mapped to CORBA interfaces. This makes logical node interaction transparent to heterogeneity in implementation (operating systems, computers, networks). CORBA does not deal with specific object functionality but with how interfaces are specified, used and managed across a system. IEC-61850 Functions are provided in the form of collaborations by means of object patterns (Gamma et al., 1995).

The interoperation is achieved based on the support of a common abstract interface (the ACSI (IEC TC-57, 2000b)) that is used to interchange information and control actions. This abstract interface is mapped to standardized communication protocols that provide the real substrate for the implementation.

The DOTS Consortium has been working since Jan 2000 and the project will end in Dec 2001. The implementation supports control objects (as specified by IEC 61850) in Eliop's remote terminal units that interoperate using SCILabs real-time object request broker (Sanz et al., 1999b).

By the time of the congress the demonstration application will be in operation in a pilot telecontrol system performing control and protection functions. It is going to be installed in an electric substation in Spain in order to demonstrate the validity of the approach under real world conditions.

However, the project results can be also applied in other industrial contexts such as water and gas utilities and, in a medium term, they could be transferred to the transport and chemical sectors 


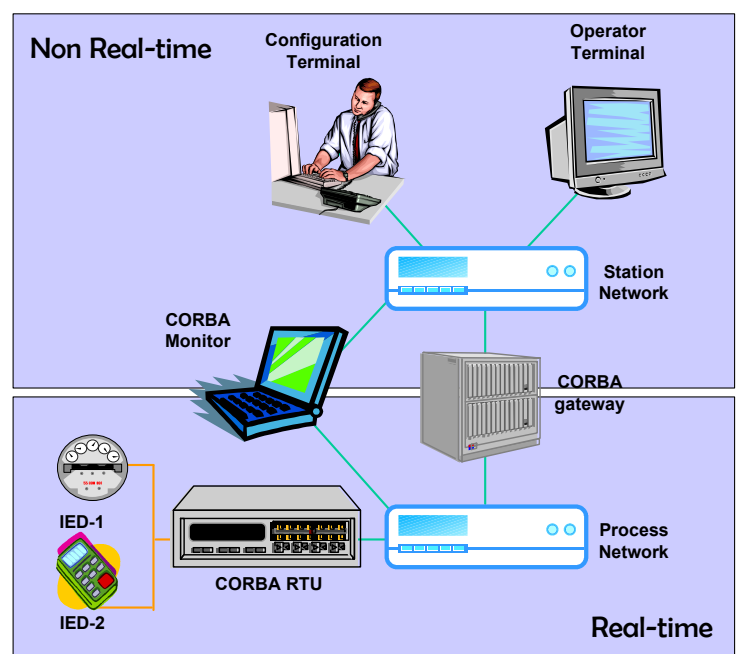

Fig. 2. DOTS architecture enables the coexistence of real-time and non real-time subsystems by means of CORBA real-time interoperability.

where integrational problems are very similar to the ones addressed by the DOTS project.

\section{INNOVATION}

The implementation of IEC 61850 over a CORBA middleware offers extremely good perspectives for complex systems construction and deployment, tasks that are quite more complex using other types of technologies.

The main point, however, is the advantages obtained for maintenance. CORBA enables running application analysis and monitorization and simplifies system evolution. Changing platforms (hardware or operating systems) is not a problem for CORBA based applications because it has been designed to provide transparency across heterogeneous systems boundaries.

From our perspective, this is the platform best suited for distributed systems construction because it provides a good mixture of performance, resource consumption and provides a good support in early phases of systems engineering lifecycles. It enormously simplifies the mapping from designs to real implementations.

\section{PROJECT RESULTS}

In accordance with the original plan of the project, the main results obtained are the following:

- A contribution to the dissemination and adoption of the IEC-61850 standard over CORBA middleware by means of two public documents: DOTS General Model Definition and DOTS Specific Communication Service
Mapping - Mapping to CORBA. Both document describe the DOTS approach to deploy 61850 applications and a concrete specification on how to do this (to be included as a part of the IEC 61850 standard).

- Apart from specfications, DOTS also produced SAS and CORBA software results:

- Embedded Software to be run on an IED $^{2}$ and in a conventional RTU ${ }^{3}$. This software implements part of theIEC61850 objects and messages of relevance to these devices.

- Real-time ORB Middleware, specifically adapted to the constraints that the telecontrol world imposes, in accordance with the IEC-61850 standard.

- System Configurator tool. A software tool for off-line definition and configuration of SAS applications based on plant topology.

- Operator Interface tool. A tool for on-line management of a running SAS.

Apart from these results, the DOTS Consortium was committed to demonstrate the validity of the approach in a pilot system 3 installed at the enduser site (Red Eléctrica de España), incorporating the software and tools developed within the project. The demonstration application was composed by:

- A deeply embedded device, acquiring three voltage and three current measures and calculating phasors and frequency from them, at the bay level supporting the following IEC61850 functions: self-checking, configuration and maintenance, control, protection, o and distributed process automation.

- A RTU at the substation level, including the following IEC-61850 functions: network management, configuration and maintenance, alarms and events management and station interlocking.

- Another RTU including extended capabilities, like still-alive image acquisition, compression and transmission for surveillance purposes.

- An Operator Interface tool for local or remote inspection and interaction with objects distributed in the power substation.

\footnotetext{
2 Integrated Electronic Device: a field device performing some specific function inside the controlled plant.

3 Remote Terminal Unit: A field computer running application specific software. The main difference with IEDs is adaptability; RTUs can be re-programmed to perform different functions; IEDs typically perform the same function during their lifespan. The border between IEDs and RTUs is getting fuzzier due to the progressive availability of small, cheap, embeddable systems.
} 


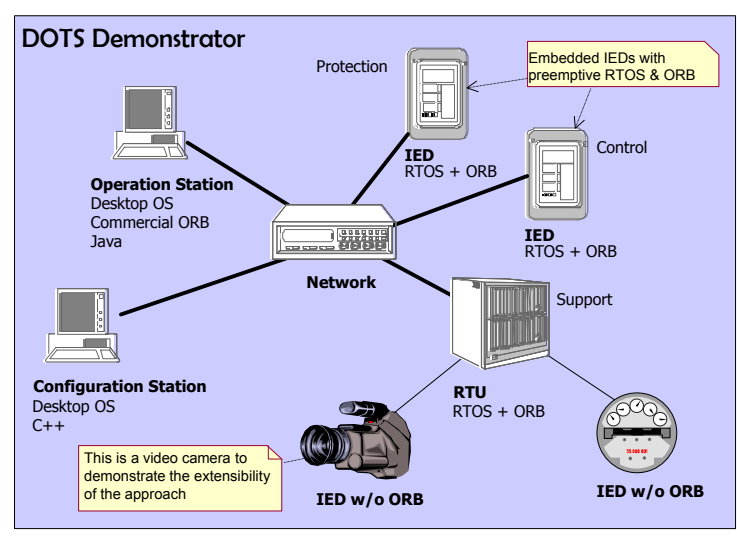

Fig. 3. DOTS demonstrator includes different types of systems as RTUs with real time operating systems and ORBs and IEDs that can include or not object request brokers.

- A System Configurator tool, working off-line, to provide interoperability at the substation configuration level.

- Real-time CORBA middleware for communications among the existing distributed objects, using serial and Ethernet links.

User interfaces of the configuration and operation tools are shown in Figure 4.

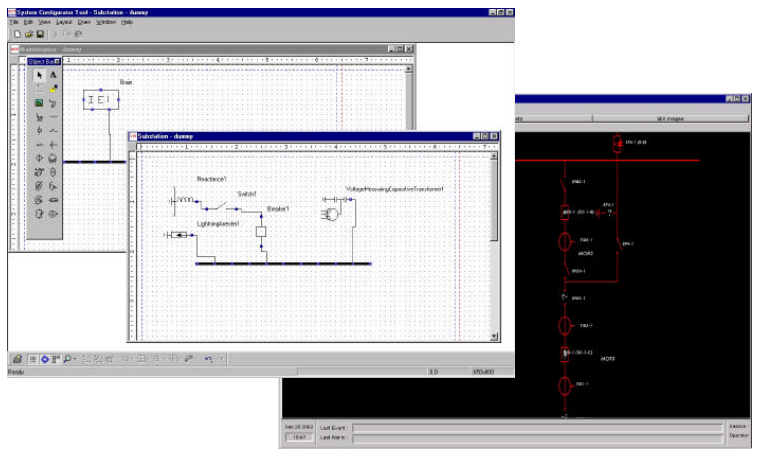

Fig. 4. User interfaces of the Operator Interface and System Configurator tools.

From our perspective, this is the standard platform best suited for distributed systems construction because it provides a good mixture of performance, resource consumption and good support in early phases of systems engineering life-cycles. It enormously simplifies the mapping from designs to real implementations.

\section{CONCLUSIONS}

Distributed control systems are complex, realtime applications that are used to monitor and control industrial processes. These applications are typically composed by a collection of software subsystems of different origins that run on heterogeneous platforms and communication systems. Examples can be found everywhere from air traffic control, to refinery control or electric utility management.

Software complexity in large control systems is enormous and hence good software tools and practices are critical.

Construction, management and maintenance of these systems is a big challenge that, in many cases, limits the evolvability of the systems. In a typical, real process control system it is possible to find systems that span several generations of technology: from computers, operating systems and languages to databases, user interfaces or communication protocols. Maintenance difficulties are so high that deep modifications most of the time imply total replacement of complete subsystems.

CORBA-based implementations of control systems open new perspectives for advanced control, because they ease the development of new functionality and enhance evolvability of applications.

This paper has presented the DOTS project objectives and results. This is a project focused on the mapping of an emergent specification for substation automation (the IEC 61850 standard) into CORBA middleware.

DOTS project results do match present technological user requirements in the SAS field (Doussin et al., 2002):

- Elimination of proprietary protocols

- Multi vendor equipment acquisition

- Cost reduction

- Simplified multi-vendor equipment integration

- Simplified training for new equipment

The system described in this paper has a real implementation in a demonstration application in an electrical substation in Spain.

And, while DOTS project results have been tested in a very specific application field (namely SAS) they can also be applied to other industrial contexts such as water and gas utilities and, in a medium term, they could be transferred to the transport and chemical sectors where integration problems are very similar to the ones addressed by the DOTS project.

\section{REFERENCES}

DOTS Consortium (1999). DOTS project programme. Technical report. CEU IST.

Doussin, Marie-Hélène, Ignacio González, JoãoPaulo Barros, Ricardo Sanz, Miguel José Segarra and Antonio Zangrilli (2002). Standardized interoperation in electrical substation automation systems. In: Third European Systems Engineering Conference, 21-24 May. INCOSE. Toulouse, France. 
Gamma, Erich, Richard Helm, Ralph Johnson and John Vlissides (1995). Design Patterns. Elements of Reusable Object Oriented Software. Addison-Wesley. Reading, MA.

IEC TC-57 (2000a). Communication networks and systems in substations - part 7-1: Basic communication structure for substation and feeder equipment - principles and models. Draft Standard 61850-7-1. IEC.

IEC TC-57 (2000b). Communication networks and systems in substations - part 7-2: Basic communication structure for substation and feeder equipment - abstract communication service interface (acsi). Draft Standard 618507-2. IEC.

IEC TC-57 (2000c). Communication networks and systems in substations part 1: Basic principles. Draft Standard 61850-1. IEC.

OMG (2000). Common object request broker architecture and specification. Release 2.4. Technical report. Object Management Group. Falls Church, USA.

Sanz, Ricardo and Fernando Matía (2000a). DOTS CORBA specific communication service mapping. External Deliverable 3.1. DOTS Consortium.

Sanz, Ricardo and Fernando Matía (2000b). DOTS general model definition. External Deliverable 1.2. DOTS Consortium.

Sanz, Ricardo, Fernando Matía and Eugenio A. Puente (1999a). The ICa approach to intelligent autonomous systems. In: Advances in Autonomous Intelligent Systems (Spyros Tzafestas, Ed.). Chap. 4, pp. 71-92. Microprocessor-Based and Intelligent Systems Engineering. Kluwer Academic Publishers. Dordretch, NL.

Sanz, Ricardo, Miguel J. Segarra, Angel de Antonio and José A. Clavijo (1999b). ICa: Middleware for intelligent process control. In: IEEE International Symposium on Intelligent Control, ISIC'1999. Cambridge, USA. 